\title{
Sortase A-Mediated Metabolic Enzyme Ligation in Escherichia coli
}

Takuya Matsumoto, ${ }^{\dagger}$ Kou Furuta, ${ }^{+}$Tsutomu Tanaka, ${ }^{\ddagger}$ and Akihiko Kondo ${ }^{+,+, *}$

${ }^{\dagger}$ Graduate School of Science, Technology and Innovation, Kobe University, 1-1

Rokkodaicho, Nada, Kobe 657-8501, Japan

Department of Chemical Science and Engineering, Graduate School of Engineering,

Kobe University, 1-1 Rokkodaicho, Nada, Kobe 657-8501, Japan

*Corresponding author;

email: akondo@kobe-u.ac.jp

tel: $+81-78-803-6196$ 


\section{Table 1. Primers used in this study}

\begin{tabular}{|c|c|}
\hline Primer name & Sequence $5^{\prime}-3^{\prime}$ \\
\hline pHLA XhoI for & CTCGAGAAGCTTGGCTGTTTTGGCGGATGA \\
\hline pHLA BglII re & AGATCTCCTTTTTCCAGAAGTGTGAAAATT \\
\hline BglII pflB for & GGAAAAAGGAGATCTATGTCCGAGCTTAATGAAAAGTTAGCCACA \\
\hline pflB LPXTG FLAG EcoRI RBS re & TTTCTCCTCTTTAATGAATTCTTACTTATCGTCGTCATCCTTGTAATCGCCACCAGTTTCCGGCAGCATAGATTGAGT \\
\hline RBS kpnI GGGGS pta for & ATTAAAGAGGAGAAAGGTACCGTGGGCGGCGGGGCCTCATCCCGTATTATTATG \\
\hline pta CMYC XhoI re & GCCAAGCTTCTCGAGTTACAGGTCCTCCTCTGAGATCAGCTTCTGCTCCTGCTGCTGTGCAGA \\
\hline pflB XhoI re & GCCAAGCTTCTCGAGTTACATAGATTGAGTGAAGGTACGAGTAAT \\
\hline XhoI pZA23 for & CTCGAGAAATTTATCAAAAAGAGTGTTGAC \\
\hline pZA23 AatII re & GACGTCGGAATTGCCAGCTGGGGCGCCCTC \\
\hline pZS4Int AatII lacI & GGCAATTCCGACGTCTAAGAAACCATTATTATCATGACA \\
\hline pZS4Int XbaI lacI re & GATAAATTTCTCGAGTCTAGACATATGAATTCGGGGCGGGATTTC \\
\hline pZA23 KpnI SrtA for & GAGGAGAAAGGTACCatgCAAGCTAAACCTCAAATTCCGAAAGATAAA \\
\hline pZA23 HindIII SrtA re & TTCGATATCAAGCTTTTATTTGACTTCTGTAGCTACAAAGATTTT \\
\hline pZA23 MCS HindIII for & AAGCTTGATATCGAATTCCTGCAGCCCGGG \\
\hline pZA23 MCS KpnI re & GGTACCTTTCTCCTCTTTAATGAATTCTGT \\
\hline pflB deple for & ATGTCCGAGCTTAATGAAAAGTTAGCCACAGCCTGGGAAGGTTTTACCAAAATTAACCCTCACTAAAGGGCG \\
\hline pflB deple re & TTACATAGATTGAGTGAAGGTACGAGTAATAACGTCCTGCTGCTGTTCTTTAATACGACTCACTATAGGGCTC \\
\hline pflb del confirm for & AGCGGTTTTGAGCACAGTAT \\
\hline pflB del confirm re & AATACAGGCAGCGCATCAGG \\
\hline pta deple for & GTGTCCCGTATTATTATGCTGATCCCTACCGGAACCAGCGTCGGTCTGACAATTAACCCTCACTAAAGGGCG \\
\hline pta deple re & TTACTGCTGCTGTGCAGACTGAATCGCAGTCAGCGCGATGGTGTAGACGATAATACGACTCACTATAGGGCTC \\
\hline pta del confirm for & CGCTGTTGTATTCACTGGTG \\
\hline pta del confirm re & CGGAACTACCCAGGTGGCAA \\
\hline poxB del for & ATGAAACAAACGGTTGCAGCTTATATCGCCAAAACACTCGAATCGGCAGGAATTAACCCTCACTAAAGGGCG \\
\hline poxB del re & TTACCTTAGCCAGTTTGTTTTCGCCAGTTCGATCACTTCATCACCGCGTCTAATACGACTCACTATAGGGCTC \\
\hline poxB confirm for & CCGGTTGTCGCTGCCTGCCG \\
\hline poxB confirm re & TTCAAACAGATAGTTATGCG \\
\hline SrtAsa C184A for & CAATTAACATTAATTACTgcgGATGATTACAATGAAAAGACAGGC \\
\hline SrtAsa C184A re & GCCTGTCTTTTCATTGTAATCATCcgcAGTAATTAATGTTAATTG \\
\hline
\end{tabular}




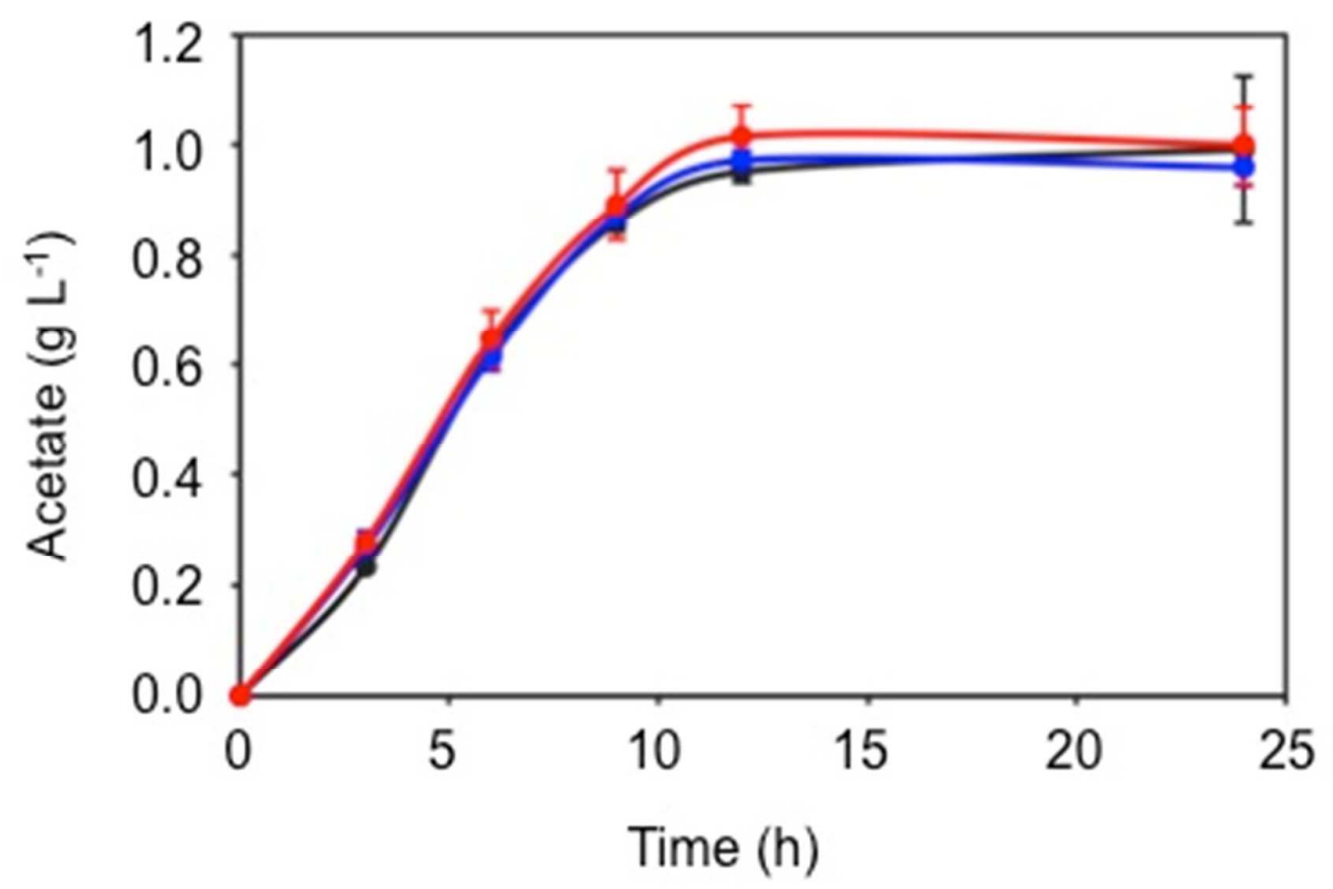

Figure S1. Effect of the time point of addition of IPTG on acetate production. The cells were inoculated at initial OD600 $=0.1$ without the addition of IPTG (light blue) or IPTG was added after $6 \mathrm{~h}$ (green blue) or $9 \mathrm{~h}$ (blue) cultivation. Data are presented as the average of three independent experiments, and error bars represent the standard deviation. 

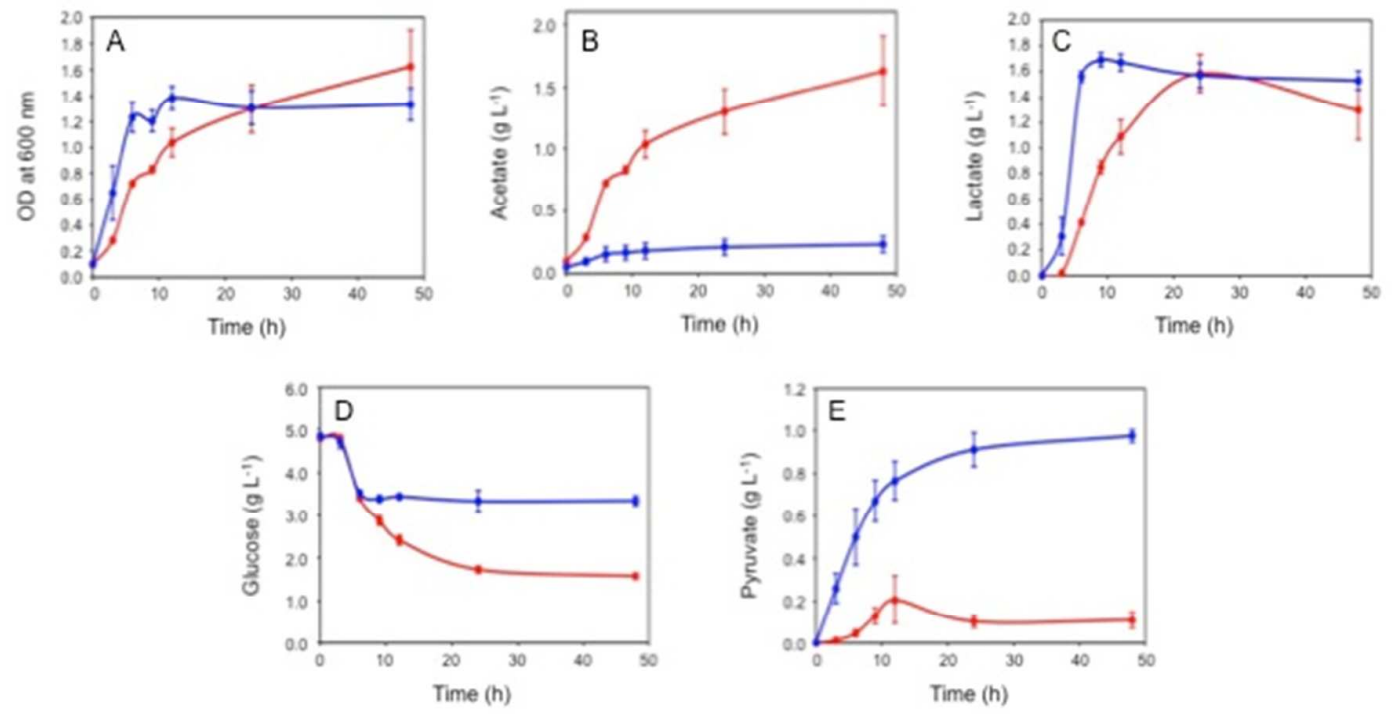

Figure S2. The cell growth (optical density at $600 \mathrm{~nm}$ ) (A), the production of acetate (B), lactate (C), pyruvate (D) and the consumption of glucose (E). The cells were inoculated at initial $O D_{600}=0.1$ and IPTG was added after 6 h cultivation. Symbols represent: KT195: red; and KT198: blue. Data are presented as the average of three independent experiments, and error bars represent the standard deviation. 